OPEN ACCESS

Edited by:

Liang Li,

Tsinghua University, China

Reviewed by:

Xiaokun Liang,

Shenzhen Institutes of Advanced

Technology, Chinese Academy of

Sciences (CAS), China

Baodong Liu,

Chinese Academy of Sciences (CAS),

China

*Correspondence:

Yangbo Ye

yangbo-ye@uiowa.edu

tThese authors have contributed equally to this work and share first

authorship

Specialty section:

This article was submitted to

Medical Physics and Imaging,

a section of the journal

Frontiers in Physics

Received: 17 March 2021

Accepted: 16 April 2021

Published: 04 June 2021

Citation:

Li X, Zhang Y, Mao S, Zhu J and Ye Y (2021) Implementation of a

Framelet-Based Spectral

Reconstruction for Multi-Slice Spiral

CT. Front. Phys. 9:682152.

doi: 10.3389/fphy.2021.682152

\section{Implementation of a Framelet-Based Spectral Reconstruction for Multi-Slice Spiral CT}

\author{
Xin $\mathrm{Li}^{1 \dagger}$, Yanbo Zhang ${ }^{2 \dagger}$, Shuwei Mao ${ }^{2,3}$, Jiehua $\mathrm{Zhu}^{4 \dagger}$ and Yangbo $\mathrm{Ye}^{5,6 *}$ \\ ${ }^{1}$ School of Mathematics, Shandong University, Jinan, China, ${ }^{2}$ Cheeloo College of Medicine, Shandong University, Jinan, \\ China, ${ }^{3}$ Shandong Public Health Clinical Center, Jinan, China, ${ }^{4}$ Department of Mathematical Sciences, Georgia Southern \\ University, Statesboro, GA, United States, ${ }^{5}$ Department of Mathematics, University of lowa, lowa City, IA, United States, \\ ${ }^{6}$ Advanced Medical Research Institute, Shandong University, Jinan, China
}

Spectral CT utilizes spectral information of X-ray sources to reconstruct energy-resolved $X$-ray images and has wide medical applications. Compared with conventional energy-integrated CT scanners, however, spectral CT faces serious technical difficulties in hardware, and hence its clinical use has been expensive and limited. The goal of this paper is to present a software solution and an implementation of a framelet-based spectral reconstruction algorithm for multi-slice spiral scanning based on a conventional energy-integrated CT hardware platform. In the present work, we implement the framelet-based spectral reconstruction algorithm using compute unified device architecture (CUDA) with bowtie filtration. The platform CUDA enables fast execution of the program, while the bowtie filter reduces radiation exposure. We also adopt an order-subset technique to accelerate the convergence. The multi-slice spiral scanning geometry with these additional features will make the framelet-based spectral reconstruction algorithm more powerful for clinical applications. The method provides spectral information from just one scan with a standard energy-integrating detector and produces color CT images, spectral curves of the attenuation coefficient at every point inside the object, and photoelectric images, which are all valuable imaging tools in cancerous diagnosis. The proposed algorithm is tested with a Catphan phantom and real patient data sets for its performance. In experiments with the Catphan 504 phantom, the synthesized color image reveals changes in the level of colors and details and the yellow color in Teflon indicates a special spectral property which is invisible in regular CT reconstruction. In experiments with clinical images, the synthesized color images provide some extra details which are helpful for clinical diagnosis, for example, details about the renal pelvis and lumbar join. The numerical studies indicate that the proposed method provides spectral image information which can reveal fine structures in clinical images and that the algorithm is efficient regarding to the computational time. Thus, the proposed algorithm has a great potential in practical application.

Keywords: spectral CT, CUDA, bowtie filtration, iterative reconstruction, multi-slice spiral CT, framelet 


\section{INTRODUCTION}

In conventional computed tomography (CT) reconstruction algorithms, X-rays are assumed to be monochromatic, thus the attenuation coefficients of objects are independent of the X-ray energy and determined by the material only. However, X-rays are polychromatic [1]. Low-energy photons are easier to be absorbed than high-energy photons. Ignoring the spectrum of $\mathrm{X}$-rays in reconstruction causes beam-hardening artifacts $[2,3]$ which are not satisfactory for clinical diagnosis. On the other hand, using energy information in polychromatic X-rays could provide properties of the material being scanned, such as the density and atomic number.

Spectral CT, which uses multiple energies of X-rays, has attracted much attention from both clinical physicists and researchers since last decade when the technology became possible. Spectral imaging can provide energyrelated attenuation characteristics of the composition of the material and quantitative tissue information to help diagnosis. Furthermore, the radiation dose is reduced since the detection and characterization of findings can be performed with spectral CT in a single exam. So spectral CT has its benefits in enhancing the accuracy of clinical diagnosis and efficiency of patient care. The basis of spectral CT is that a CT scan can be decomposed into a set of multiple basis materials if the projection data is measured at different energies [4]. This leads to the development of dual-energy CT and multi-spectral CT [5]. Dual-energy refers to the use of two X-ray energies in scanning. Multi-spectral CT is an extension of dual-energy CT. Currently dual-energy CT is the most common form of spectral CT used in clinical applications due to the limit of technology [6].

There are different technologies to utilize dual-energy CT but mainly in two categories. One is source-based, and the other one is detector-based. The first category utilizes X-ray beams with different energies by employing two X-ray tubes with different potentials or a single X-ray tube switching between low and high potentials $[7,8]$. The switch occurs during the collection of projection data in a single scan or after a full projection scan in two scans. The second category utilizes energy-discriminative detectors in a single X-ray spectrum scan [9]. This involves duallayer detectors with a different $\mathrm{X}$-ray stopping power in each layer or a photon-counting detector. Each method of dual-energy CT has its respective advantages and disadvantages [10]. Some spectral CT iterative reconstruction algorithms are developed for the hardware technology methods [11-13].

In either source based or detector based methods, spectral CT needs more complicated equipment for data requisition and requires longer time for data requisition and image reconstruction than conventional CT. Besides, for a hospital, adding a new spectral CT scanner to its lab which has facilitated a conventional CT scanner involves practical issues in cost and space. Recently, a framelet-based iterative reconstruction algorithm is proposed to solve the spectral CT reconstruction problem from the aspect of software in the current CT hardware platform [14-18] by our group. The key technique is the sparse representation of X-ray attenuation coefficients in a framelet system. The method provides spectral information from just one scan with a standard energy-integrating detector and produces color CT images, spectral curves of the attenuation coefficient at every point inside the object, and photoelectric images, which are all valuable imaging tools in cancerous diagnosis.

In this paper, we extend the framelet-based spectral reconstruction algorithm from fan-beam geometry to multi-slice spiral scanning and implement the algorithm using compute unified device architecture (CUDA) with bowtie filtration. The platform CUDA allows fast execution of the program, while the bowtie filter reduces the radiation exposure by shaping an X-ray beam. Therefore, the multi-slice spiral scanning geometry with these additional features will make the framelet-based spectral reconstruction algorithm more powerful for clinical applications.

The rest of the paper is organized as follows. In section 2 , we describe the methodology and the implementation of the framelet-based iterative reconstruction algorithm for multislice spiral scanning. In section 3, the results from extensive experiments on a Catphan phantom and real patient datasets are summarized. Discussions and conclusions are given in section 4 .

\section{METHOD}

\subsection{Methodology}

In this section we extend the framelet-based spectral reconstruction algorithm from fan-beam geometry [14-18] to multi-slice spiral scanning with the bowtie filtering making it more practical in clinical applications.

Let $\mu(r, E)$ be the X-ray attenuation coefficient at point $r \in \Omega$ for energy level $E$, where $\Omega$ is the image domain. The attenuation coefficient can be approximated by [19].

$$
\mu(r, E)=\phi(r) E^{-3}+\theta(r) f_{K N}(E),
$$

where the photoelectric component $\phi(r)$ and the Compton scatter component $\theta(r)$ are independent of energy $E$, and the Klein-Nishina function $f_{K N}(E)$ is given by

$$
\begin{aligned}
f_{K N}(E)= & \frac{1+\varepsilon}{\varepsilon^{2}}\left(\frac{2(1+\varepsilon)}{1+2 \varepsilon}-\frac{1}{\varepsilon} \ln (1+2 \varepsilon)\right) \\
& +\frac{1}{2 \varepsilon} \ln (1+2 \varepsilon)-\frac{1+3 \varepsilon}{(1+2 \varepsilon)^{2}},
\end{aligned}
$$

with $\varepsilon=E / 511 \mathrm{keV}$.

We consider a polychromatic acquisition model in this paper. Let $L$ be an X-ray beam and $I_{0}(E)$ be the known spectrum of the $\mathrm{X}$-ray tube. The intensity measured by a detector bin after the beam $L$ passes through the image is

$$
I=\int_{0}^{\infty} I_{0}(E) \exp \left(-\int_{L} \mu(r, E) d r\right) d E .
$$

In our previous work $[14,15], I_{0}(E)$ is constant for all X-ray beams. Here we assume $I_{0}(E)$ varies with respect to positions of X-ray beams and take bowtie filtering into consideration. Then the $\mathrm{x}$-ray spectrum distribution after passing the bowtie filtering is

$$
I_{0}(E, L)=I_{0}(E) \exp \left(-\mu_{\text {bowtie }}(r, E) t(L)\right),
$$


where $\mu_{\text {bowtie }}$ indicates the linear attenuation coefficient of the bowtie material (e.g., aluminum), and $t(L)$ denotes the bowtie thickness along the X-ray path $L$.

Due to the relative position relationship of the X-ray tube, the bowtie filter, and the detector, the bowtie filter and the detector are fixed during a scan, and the distribution of filtered X-ray spectrum are also fixed on the detector. Let $d_{i, j}$ be the detector bin at the position $(i, j)$ which receives the X-ray beam $L$. Then Equation (4) can be rewritten as

$$
I_{0}\left(E, d_{i, j}\right)=I_{0}(E) \exp \left(-\mu_{\text {bowtie }}(r, E) t\left(d_{i, j}\right)\right) .
$$

Consequently, Equation (3) can be expressed as

$$
I=\int_{0}^{\infty} I_{0}\left(E, d_{i, j}\right) \exp \left(-\int_{L} \mu(r, E) d r\right) d E .
$$

In the discrete setting, we denote the photon energies by $E_{k}, k=$ $1,2, \ldots, K$, the observed measurements by $y_{i}, i=1,2, \ldots, M$, and the linear attenuation coefficients by $\mu_{j k}, j=1,2, \ldots, J$, and $k=1,2, \ldots, K$, where $i, j, k$ are indexes of the detector, pixel, and energy, respectively. Then Equation (1) can be rewritten as

$$
\mu_{j k}=\phi_{j} \Phi\left(E_{k}\right)+\theta_{j} \Theta\left(E_{k}\right)
$$

where $\Phi\left(E_{k}\right)=E_{0}^{3} / E_{k}^{3}$ for a reference energy $E_{0}$, and $\Theta\left(E_{k}\right)=f_{K N}\left(E_{k}\right) / f_{K N}\left(E_{0}\right)$. Note that $\phi_{j}$ and $\theta_{j}$ are unknowns, representing photoelectric and Compton scatter terms at energy $E_{0}$, respectively. Substituting Equation (7) into (6), we get an acquisition model

$$
\hat{y}_{i}=\sum_{k=1}^{K} b_{i k} \exp \left(-\sum_{j=1}^{J} l_{i j}\left(\phi_{j} \Phi\left(E_{k}\right)+\theta_{j} \Theta\left(E_{k}\right)\right)\right)
$$

where $b_{i k}$ is the total intensity detected by detector at the ith projection and the kth energy. The measured data $y_{i}$ approximately follow a Poisson distribution [20] with expectation $\hat{y}_{i}$, where the probability density function is

$$
p\left(y_{i}\right)=\frac{\hat{y}_{i}^{y_{i}}}{y_{i} !} \exp \left(-\hat{y}_{i}\right)
$$

The log-likelihood is given by

$$
L(\phi, \theta)=\sum_{i=1}^{M}\left(y_{i} \log \left(\hat{y}_{i}\right)-\hat{y}_{i}\right)
$$

The minimization problem becomes

$$
\min _{\phi, \theta}-L(\phi, \theta), \text { subject to image sparsity of } \phi \text { and } \theta \text {. }
$$

For the sparsity constraint, we use the discrete framelet transform given by [14]

$$
D=\left[\begin{array}{c}
h_{0} * \\
\vdots \\
h_{s} *
\end{array}\right]
$$

where $h_{i} *, i=0,1, \ldots, s$, is the matrix form of the discrete convolution with kernel $h_{i}$. In this work, we apply the following filters in bivariate Harr wavelet framelet system:

$$
\begin{aligned}
& h_{0}=\frac{1}{4}\left[\begin{array}{ll}
1 & 1 \\
1 & 1
\end{array}\right], h_{1}=\frac{1}{4}\left[\begin{array}{ll}
1 & -1 \\
1 & -1
\end{array}\right], \\
& h_{2}=\frac{1}{4}\left[\begin{array}{cc}
1 & 1 \\
-1 & -1
\end{array}\right], h_{3}=\frac{1}{4}\left[\begin{array}{cc}
1 & -1 \\
-1 & 1
\end{array}\right] .
\end{aligned}
$$

To solve the optimization problem (11), the coefficients $\phi$ and $\theta$ should be non-negative and sparse in the framelet domain. Therefore, the iterative reconstruction can be split into the following three steps.

(1) Data fidelity:

$$
\left\{\begin{array}{l}
\phi_{j}^{n+\frac{1}{3}}=\phi_{j}^{n}-\delta_{1} \frac{\partial L}{\partial \phi_{j}}\left(\phi^{n}, \theta^{n}\right)\left(\sum_{h=1}^{J}\left(\frac{\partial^{2} L}{\partial \phi_{j} \partial \phi_{h}}+\frac{\partial^{2} L}{\partial \phi_{j} \partial_{h}}\right)\left(\phi^{n}, \theta^{n}\right)\right)^{-1} \\
\theta_{j}^{n+\frac{1}{3}}=\theta_{j}^{n}-\delta_{2} \frac{\partial L}{\partial \theta_{j}}\left(\phi^{n}, \theta^{n}\right)\left(\sum_{h=1}^{J}\left(\frac{\partial^{2} L}{\partial \theta_{j} \partial \phi_{h}}+\frac{\partial^{2} L}{\partial \theta_{j} \partial \theta_{h}}\right)\left(\phi^{n}, \theta^{n}\right)\right)^{-1}
\end{array}\right.
$$

(2) Nonnegativity:

$$
\left\{\begin{array}{l}
\phi_{j}^{n+\frac{2}{3}}=\max \left\{\phi_{j}^{n+\frac{1}{3}}, 0\right\} \\
\theta_{j}^{n+\frac{2}{3}}=\max \left\{\theta_{j}^{n+\frac{1}{3}}, 0\right\} .
\end{array} .\right.
$$

(3) Sparsity:

$$
\left\{\begin{array}{l}
\phi_{j}^{n+1}=W^{T} T_{\lambda_{1}}\left(W \phi_{j}^{n+\frac{2}{3}}\right) \\
\theta_{j}^{n+1}=W^{T} T_{\lambda_{2}}\left(W \theta_{j}^{n+\frac{2}{3}}\right) .
\end{array}\right.
$$

where $W$ denotes the tight frame analysis transform, and $T_{\lambda}$ is a soft-thresholding operator, defined as $T_{\lambda}(x)=\frac{|x|-\lambda}{|x|} x$ if $|x|>\lambda$, otherwise $T_{\lambda}(x)=0$.

\subsection{Algorithm and Implementation}

We implement our method for multi-slice spiral scanning using both Matlab and CUDA. CUDA is a software development platform enabling general purpose C-like programs on the NVIDIA graphics processing unit (GPU) [21]. Since the operations of forward and backward projections are time consuming, we use CUDA in GPU card to perform forward and backward projections. Matlab is user-friendly for fast development of new methods, so we conduct non-negativity and sparsity constraints on Matlab and sync the results from CUDA with the embedded "mex" functions in Matlab".

To accelerate the convergence, we adopt the ordered-subset (OS) technique [22]. We uniformly divide total projections into 16 subsets and update photoelectric and Compton scatter volumes on every subset's iteration. The reconstruction stops after a preset number of iterations, which is set to 10 in this study.

Our algorithm for spiral scanning spectral reconstruction is summarized as follows.

${ }^{1}$ https://www.mathworks.com/help/parallel-computing/accessing-advancedcuda-features-using-mex.html (2021). 


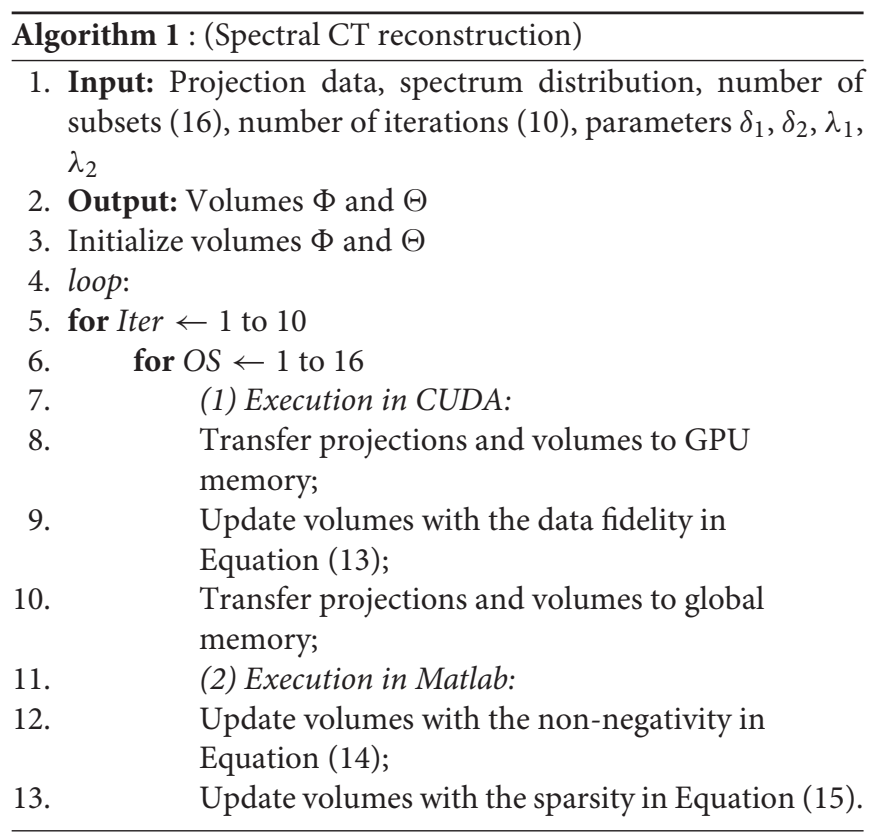

\section{RESULTS}

In this section, the proposed algorithm is evaluated with a Catphan phantom and real patient data sets. Numerical experiments are conducted on an Xeon Gold 6242R @3.1 GHz/NVIDIA Quadro RTX 8000 workstation in Matlab R2017b \& CUDA 9.0. The spiral CT scanning has 960 projections per rotation, and the pitch is 1 . There are 16 rows of detectors and each row consists of 896 detector bins. Each reconstructed slice consists of $512 \times 512$ pixels. The slice thicknesses of reconstructed volumes are $5 \mathrm{~mm}$ for chest and abdomen, and $2.5 \mathrm{~mm}$ for head data in this work. We use the proposed algorithm to reconstruct the photoelectric coefficients $\phi$ and the Compton scatter coefficients $\theta$. In the experiments presented in this paper, we choose step size $\delta_{1}=0.5$ for $\phi, \delta_{2}=1$ for $\theta$, and threshold parameters $\lambda_{1}=1.5 \times 10^{-4}$ for $\phi, \lambda_{2}=4.5 \times 10^{-4}$ for $\theta$.

\subsection{Experiment With Catphan Phantom}

Experiments are conducted with the Catphan 504 phantom designed for multi-slice spiral CT algorithm evaluation by the Phantom Laboratory ${ }^{2}$. The image reconstructed at monochromatic $70 \mathrm{keV}$ labeled with different materials and the color image overlaid by the attenuation coefficients at monochromatic 40, 50, and $100 \mathrm{keV}$ are shown in Figure 1. From the synthesized color image, changes in the level of colors and details are visible. In specific, the yellow shown in Teflon indicates a special spectral property which is invisible in regular $\mathrm{CT}$ reconstruction. In addition, acrylic is more clear in the synthesized color image.

To evaluate the quantitative performance of the proposed method, we compute the mean values and standard deviations of CT numbers of specific materials in the reconstructed Catphan 504 phantom. Table 1 lists the mean CT numbers $\mu$ with their

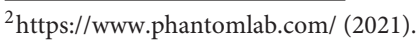

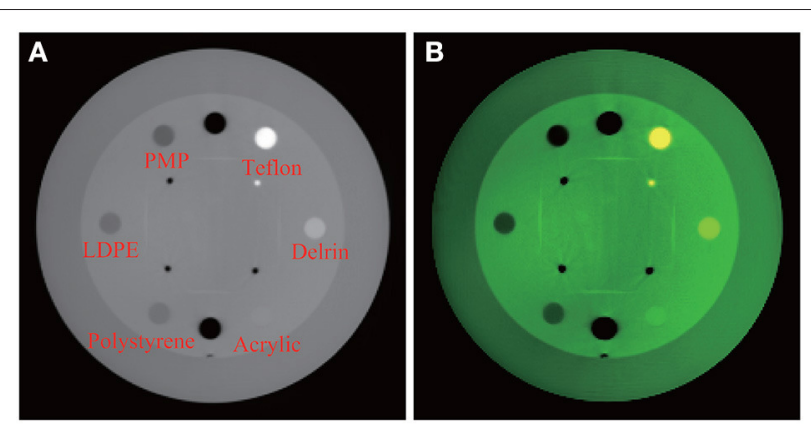

FIGURE 1 | Experiment with Catphan 504. From left, (A) reconstructed image at monochromatic $70 \mathrm{keV}$ with labels of various materials; (B) synthesized color image.

standard deviations $\sigma$ computed at monochromatic 40, 50, and $100 \mathrm{keV}$, together with reference CT numbers provided by manufacturer's manual. It is remarkable that the standard deviations $\sigma$ are relatively small in most of the cases, in the sense that the length of $95 \%$ confidence interval is much smaller than the corresponding CT number range given in the manual, except for LDPE. For example, the 95\% confidence interval $(\mu-2 \sigma, \mu+2 \sigma)$ for the CT number of polystyrene at $100 \mathrm{keV}$ is $(-72.2,-51.8)$. Its length of 20.4 is significantly smaller than 36 , the length of the corresponding interval $(-65,-29)$ in the manual. This is a manifestation of the accuracy of our spectral reconstruction algorithm.

Note that manufacturer's reference CT numbers are obtained from polychromatic X-ray systems. It is known that CT numbers depend largely on energy levels and spectra of X-ray sources [23]. Since the X-ray spectra used for Catphan reference CT numbers are not known, there are no direct ways to compare the CT numbers reconstructed by our algorithm and the manufacturer's reference CT numbers. What Table 1 shows, however, is a clear trend of dependence of CT numbers on X-ray energy levels. Except for Delrin, the $95 \%$ confidence intervals of our CT numbers overlap with the corresponding reference intervals at least at some energy levels.

\subsection{Experiment With Clinical Images}

Experiments are also conducted with clinical data. Conventional FBP reconstructed images of patients' head, chest, and abdomen images are obtained from a CT scanner XHCT-16 manufactured by Shinva Medical Instrument Limited Co. Then the images reconstructed and synthesized from the proposed algorithm are compared with the images reconstructed directly from the Shinva XHCT-16.

A brain image reconstructed directly by a Shinva XHCT-16 is shown in Figure 2A. The photoelectric, Compton scatter, and attenuation coefficients reconstructed by the proposed method at monochromatic $70 \mathrm{keV}$ from the same data are shown in Figures 2B-D. The color image overlaid by the attenuation coefficients at monochromatic 40,50, and $100 \mathrm{keV}$ is shown in Figure 3. The following features are observed from Figures 2, 3. Firstly, the images in Figures $2 \mathbf{B}-\mathbf{D}$ reconstructed by the proposed method provide more details about the brain tissue 
TABLE 1 | CT numbers reconstructed for materials in Catphan 504 (Unit: HU).

\begin{tabular}{|c|c|c|c|c|c|c|}
\hline Material & Polystyrene & LDPE & PMP & Teflon & Delrin & Acrylic \\
\hline $\mathrm{CT}$ numbers at $40 \mathrm{keV} \pm \mathrm{SD}$ & $-98 \pm 4.8$ & $-120 \pm 11.8$ & $-254 \pm 6.9$ & $1052 \pm 10.5$ & $415 \pm 7$ & $134 \pm 5.8$ \\
\hline CT numbers at $50 \mathrm{keV} \pm \mathrm{SD}$ & $-92 \pm 4.3$ & $-120 \pm 10.8$ & $-247 \pm 6.0$ & $1068 \pm 9.6$ & $422 \pm 6.3$ & $143 \pm 5.1$ \\
\hline $\mathrm{CT}$ numbers at $100 \mathrm{keV} \pm \mathrm{SD}$ & $-62 \pm 5.1$ & $-100 \pm 13.0$ & $-215 \pm 6.9$ & $1146 \pm 11.4$ & $469 \pm 7.3$ & $192 \pm 5.8$ \\
\hline Reference CT numbers & $-65:-29$ & $-121:-87$ & $-220:-172$ & $941: 1060$ & $344: 387$ & $92: 137$ \\
\hline
\end{tabular}
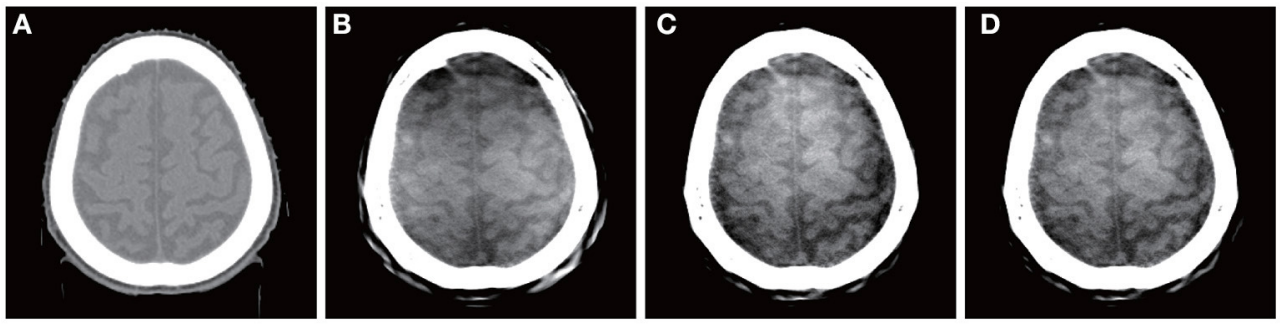

FIGURE 2 | Brain images reconstructed by a Shinva XHCT-16 and the proposed method at monochromatic 70 keV from the same data. From left, (A) Shinva XHCT-16 with the display window [-150, 210] (Unit: HU); (B) reconstructed photoelectric component; (C) reconstructed Compton scatter component;

(D) reconstructed attenuation coefficients.

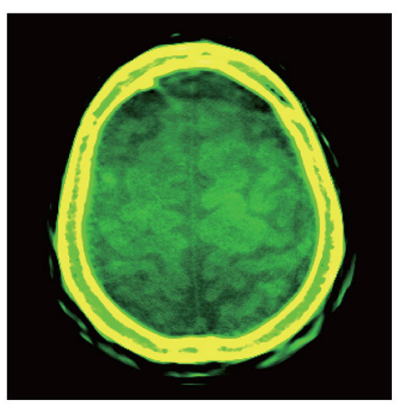

FIGURE 3 | Color image overlaid by the attenuation coefficients at monochromatic 40, 50, and $100 \mathrm{keV}$, with the display window [-150, 210] (Unit: $\mathrm{HU}$ ) of the components at each keV.

than the image Figure 2A from the Shinva XHCT-16. For example, there is no structure except noise displayed in the brain tissue ditches in Figure 2A, but some details are visible in the brain tissue ditches in Figures 2B-D. Secondly, the green color of the brain tissue and the yellow color of head bones in Figure 3 indicate different spectral properties of brain tissue, skull bone, and trabecular bone. In addition to the brain tissue, Figure 3 displays the inner layer of skull bone, the outer layer of skull bone, and the trabecular bone between them, while a conventional gray CT image can hardly show them simultaneously. Thirdly, the thin green layer inside the skull bone shows clearly the connection between meninges and the brain tissue ditches, which is not indicated in Figure 2A anywhere. The upper sagittal sinus connecting the central longitudinal fissure at the back of the brain tissue displays its internal structure and its connection with the meninges, which is not shown in
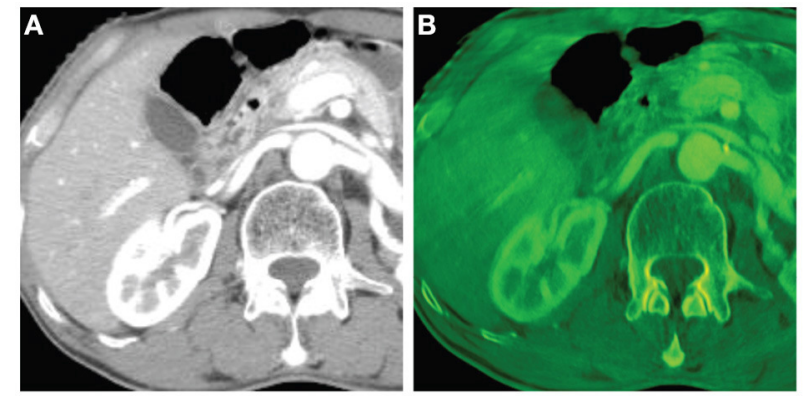

FIGURE 4 | Abdomen images with the display window [-280, 320] (Unit: HU). From left, (A) image reconstructed by a Shinva XHCT-16; (B) synthesized color image with components at $40 \mathrm{keV}, 50 \mathrm{keV}$, and $100 \mathrm{keV}$.

Figure 2A. Finally, the gray ring between the skull bone and brain tissue in Figure 2A doesn't provide any structural information, in particular, it is wide and uniformly gray in the forehead region. It is remarked that the corresponding region in Figures $2 \mathrm{~B}-\mathrm{D}, \mathbf{3}$ is informative and provides clear structural information.

Figures 4, 5 show the images reconstructed by a Shinva XHCT-16 and the synthesized color images by the proposed method, for an abdomen image and a chest image, respectively. Compared with the images by the Shinva XHCT-16, the color images provide some extra details which are helpful for clinical diagnosis, for example, details about the renal pelvis and lumbar joint in Figure 4B and the rectangular region in Figure 5B.

In summary, the proposed reconstruction algorithm provides spectral image information which reveals tiny structures in clinical images and is helpful for clinical diagnosis. 


\subsection{Computation and Convergence Speed}

In this section, we compare the computational time of the proposed algorithm with that of the fan beam spectral reconstruction algorithm [14]. Experiments with both algorithms are conducted on the Xeon Gold 6242R @3.1 GHz/NVIDIA Quadro RTX 8000 workstation in the same operating environment. Chest data are selected as an example. The reconstructed volume has 723 slices, and each slice is with three sizes of $1,024^{\star} 1,024,768^{\star} 768$, and $512^{\star} 512$ pixels, respectively. The total number of iterations is preset to 10 because it is observed that the algorithm converges rapidly before the 10th iteration but changes slightly and slowly after 10 iterations, as shown in Figure 6. The average running times of an iteration for a single slice with the previous fan beam algorithm are $1686.5,936.1$, and $393.7 \mathrm{~s}$ for the three image resolutions, respectively, while with our proposed algorithm, the average running times of an iteration for the whole volume are 1164.2, 714.1 , and $375.3 \mathrm{~s}$, respectively, for the three image resolutions. The results show that the proposed algorithm significantly reduces the computational time.
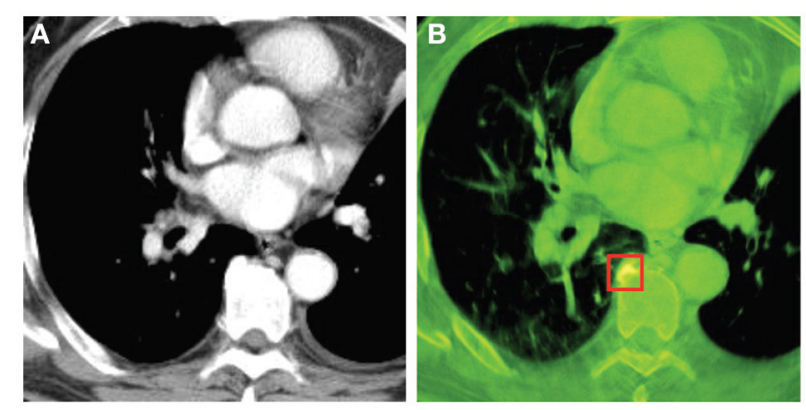

FIGURE 5 | Chest images with the display window [-532, 166] (Unit: HU). From left, (A) image reconstructed by Shinva XHCT-16; (B) synthesized color image with components at $40 \mathrm{keV}, 50 \mathrm{keV}$, and $100 \mathrm{keV}$.

\section{DISCUSSIONS AND CONCLUSIONS}

\subsection{Comparison With Existing Spectral Reconstruction Algorithms}

In our previous method [14], the reconstruction process were all realized in the environment of MATLAB and running on the CPU without any acceleration technologies. Besides, it only reconstructs a central slice from the rebined fanbeam projections. In comparison, the method proposed in this paper reconstructs a whole volume from the spiral multi-slice projections and adopts GPU to significantly speed up the reconstruction, making our method a great potential in practical application.

In De Man et al. [24], the authors select a set of base substances including air, water, bone, and iron. Using the photoelectric coefficients $\phi$ and the Compton coefficients $\theta$ of these base substances, a piecewise-linear $\phi-\theta$ curve is plotted. By assuming all other substances have their $\phi$ and $\theta$ coefficients lie on this $\phi-\theta$ curve, they effectively reduce the number of unknowns from $2 J$ to $J$ after discretization as in Equation (7). De Man et al. [24] proposes an iterative maximum-likelihood algorithm (IMPACT) to reconstruct these $J$ unknowns and a postreconstruction algorithm (IBHC) for beam-hardening correction.

As opposite to [24], the algorithm presented in this paper is a true spectral reconstruction in the sense of solving for all $2 J$ unknowns. We do not have codes for IMPACT and IBHC to run on our datasets to make a direct comparison, but by looking at [24, Figures 5-9] and our Figures 1-5, our reconstructions appear better.

\subsection{Limitations}

State-of-the-art spectral CT platforms such as the detectorbased IQon Elite Spectral CT by Philips ${ }^{3}$, dual-energy spectral CT Discovery $750 \mathrm{HD}$ by $\mathrm{GE}^{4}$, and dual-source spectral CT

${ }^{3}$ https://www.usa.philips.com/healthcare/product/HC729332/iqon-elite-spectralct-ct-scanner (2021).

${ }^{4}$ https://www.gehealthcare.com/-/jssmedia/c47da2e232d1498db841631ee28eb3d9. $\operatorname{pdf}(2021)$.
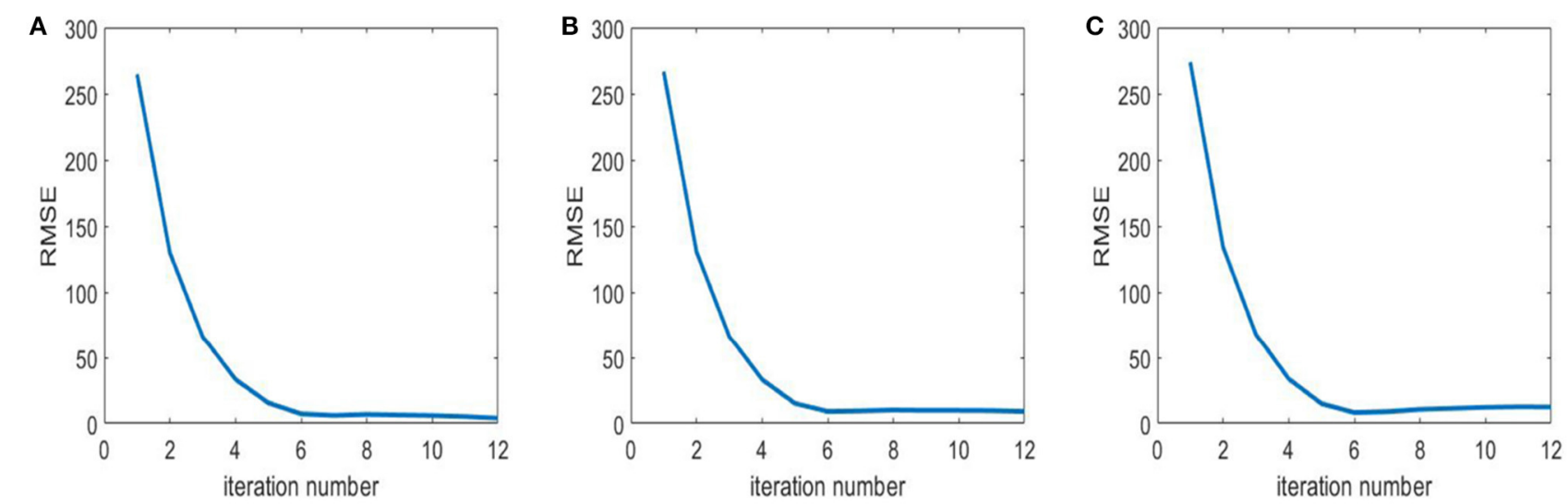

FIGURE 6 | Root Mean Square Error (RMSE) of Teflon in Figure 1 to reveal the convergence rate of the proposed algorithm. From left, (A) RMSE of Teflon under 40 kev; (B) RMSE of Teflon under $50 \mathrm{kev;}$ (C) RMSE of Teflon under $100 \mathrm{kev}$. 
SOMATOM Force by Siemens $s^{5}$ use sophisticated hardware technology and produce spectral CT images of high quality. The algorithm presented here is on the other hand a software solution to spectral CT. Since our algorithm is based on datasets obtained from conventional energy-integrated CT scanners, the amount of information in our scanned data is much less than those in footnotes 3,4 , and 5 . This determines a priori that our image quality cannot be expected to reach the levels of footnotes 3,4 , and 5. Our algorithm, however, may provide a low-cost solution to spectral CT and hence promote more clinical usage.

\subsection{Conclusion}

In this paper, we implement a framelet-based spectral reconstruction for conventional multi-slice spiral CT using CUDA with bowtie filtration and the OS technique. The experiments demonstrate that the synthesized color image produced by the proposed algorithm can reveal fine structures in clinical images and that the algorithm is efficient regarding to the computational time. Thus, the proposed algorithm has a great potential in practical application. In the future, we will further investigate the proposed method in clinical settings and study the convergence of proposed algorithm in theory.

${ }^{5}$ https://www.siemens-healthineers.com/en-us/computed-tomography/dualsource-ct/somatom-force (2021).

\section{REFERENCES}

1. Brooks RA. Energy dependence of the Hounsfield number. In: Proceedings of the Annual Symposium on Computer Application in Medical Care Washington, DC (1977). p. 368-71.

2. Brooks RA, Di Chiro G. Beam hardening in X-ray reconstructive tomography. Phys Med Biol. (1976) 21:390-8. doi: 10.1088/0031-9155/21/3/004

3. Herman GT. Correction for beam hardening in computed tomography. Phys Med Biol. (1979) 24:81-106. doi: 10.1088/0031-9155/24/1/008

4. Alvarez RE, Macovski A. Energy-selective reconstructions in $\mathrm{X}$ ray computerized tomography. Phys Med Biol. (1976) 21:733-44. doi: 10.1088/0031-9155/21/5/002

5. Garnett R. A comprehensive review of dual-energy and multispectral computed tomography. Clin Imaging. (2020) 67:160-9. doi: 10.1016/j.clinimag.2020.07.030

6. Johnson T, Fink C, Schönberg SO, Reiser MF. Dual Energy CT in Clinical Practice. Berlin: Springer (2011).

7. Macovski A, Alvarez RE, Chan JL, Stonestrom JP, Zatz LM. Energy dependent reconstruction in X-ray computerized tomography. Comput Biol Med. (1976) 6:325-34. doi: 10.1016/0010-4825(76)90069-x

8. Flohr TG, Bruder H, Stierstorfer K, Petersilka M, Schmidt B, McCollough $\mathrm{CH}$. Image reconstruction and image quality evaluation for a dual source CT scanner. Med Phys. (2008) 35:5882-97. doi: 10.1118/1.3020756

9. Barnes GT, Sones RA, Tesic MM, Morgan DR, Sanders JN. Detector for dual-energy digital radiography. Radiology. (1985) 156:537-40. doi: 10.1148/radiology.156.2.4011921

10. McCollough CH, Leng S, Yu L, Fletcher JG. Dual- and multi-energy CT: principles, technical approaches, and clinical applications. Radiology. (2015) 276:637-53. doi: 10.1148/radiol.2015142631

11. Yan CH, Whalen RT, Beaupré GS, Yen SY, Napel S. Reconstruction algorithm for polychromatic CT imaging: application to beam hardening correction. IEEE Trans Biomed Eng. (2009) 19:1-11. doi: 10.1109/42.832955

12. Fessler JA, Elbakri IA, Sukovic P, Clinthorne NH. Maximum-likelihood dualenergy tomographic image reconstruction. In: Proceedings Volume 4684 Medical Imaging. Bellingham, WA (2002) p. 38-49.

13. Zhao B, Ding H, Lu Y, Wang G, Zhao J, Molloi S. Dual-dictionary

\section{DATA AVAILABILITY STATEMENT}

The datasets presented in this article are not readily available because, proprietary data will not be released. Requests to access the datasets should be directed to yangbo-ye@uiowa.edu.

\section{AUTHOR CONTRIBUTIONS}

All authors listed have made a substantial, direct and intellectual contribution to the work, and approved it for publication.

\section{FUNDING}

The authors express their sincere thanks to Shandong University for Grant No. 10000086393120 (Precision Oncology) and to Xinhua Medical Instrument Co. Ltd. (Shinva) for a grant.

\section{ACKNOWLEDGMENTS}

The authors express their sincere thanks to Shandong University for facilities and support, to Xinhua Medical Instrument Co. Ltd. (Shinva) for cooperation and clinical and experimental data, and to Analogic Corporation for a permission to scanned data. Gratitude is also due to Zhien Qin, Charles Shaughnessy, and Zhendong Wang for their valuable help.

learning-based iterative image reconstruction for spectral computed tomography application. Phys Med Biol. (2012) 57:8217-29. doi: 10.1088/0031-9155/57/24/8217

14. Wang Y, Wang G, Mao S, Cong W, Ji Z, Cai JF, et al. A framelet-based iterative maximum-likelihood reconstruction algorithm for spectral CT. Inverse Probl. (2016) 32:115021. doi: 10.1088/0266-5611/32/11/115021

15. Wang Y, Wang G, Mao S, Cong W, Ji Z, Cai JF, et al. A spectral interior CT by a framelet-based reconstruction algorithm. J X-Ray Sci Technol. (2016) 24:771-85. doi: 10.3233/XST-160586

16. Cong W, Ye Y, Wang G, Mao S, Wang Y. Devices, Systems and Methods Utilizing Framelet-Based Iterative Maximum-Likelihood Reconstruction Algorithms in Spectral CT. Alexandria, VA: The United States Patent and Trademark Office (USPTO), US Patent 10489942 (2019).

17. Cong W, Ye Y, Wang G, Mao S, Wang Y. Devices, Systems and Methods Utilizing Framelet-Based Iterative Maximum-Likelihood Reconstruction Algorithms in Spectral CT. Beijing, China: The China National Intellectual Property Administration, China Patent Application: 201780019606.1 (2017).

18. Cong W, Ye Y, Wang G, Mao S, Wang Y. Devices, Systems and Methods Utilizing Framelet-Based Iterative Maximum-Likelihood Reconstruction Algorithms in Spectral CT. Alexandria, VA: The United States Patent and Trademark Office (USPTO), US Notice of Allowance: US Patent Application: $16 / 597,250$ (2020).

19. Stonestrom JP, Alvarez RE, Macovski A. A framework for spectral artifact corrections in X-Ray CT. IEEE Trans Biomed Eng. (1981) 2:128-41. doi: 10.1109/TBME.1981.324786

20. Hsieh J. Adaptive streak artifact reduction in computed tomography resulting from excessive X-ray photon noise. Med Phy. (1998) 25:2139-47. doi: $10.1118 / 1.598410$

21. Jia X, Dong B, Lou Y, Jiang SB. GPU-based iterative cone-beam CT reconstruction using tight frame regularization. Phys Med Biol. (2011) 56:3787-807. doi: 10.1088/0031-9155/56/13/004

22. Wang G, Jiang M. Ordered-subset simultaneous algebraic reconstruction techniques (OS-SART). JX-ray Sci Technol. (2004) 12:169-77.

23. Chan MF, Yang J, Song Y, Burman C, Chan P, Li S. Evaluation of imaging performance of major image guidance systems. Biomed Imaging Interv J. (2011) 7:e11. doi: 10.2349/biij.7.2.e11 
24. De Man B, Nuyts J, Dupont P, Marchal G, Suetens P. An iterative maximumlikelihood polychromatic algorithm for CT. IEEE Trans Med Imaging. (2001) 20:999-1008. doi: 10.1109/42.959297

Conflict of Interest: Intellectual property related to International Patent Application No. PCT/US17/23851, including references [16-18], was licensed to Shandong Xinhua Medical Instrument Co. Ltd. who provided partial funding to this research.
Copyright (c) 2021 Li, Zhang, Mao, Zhu and Ye. This is an open-access article distributed under the terms of the Creative Commons Attribution License (CC BY). The use, distribution or reproduction in other forums is permitted, provided the original author(s) and the copyright owner(s) are credited and that the original publication in this journal is cited, in accordance with accepted academic practice. No use, distribution or reproduction is permitted which does not comply with these terms. 